IDDF2018-ABS-0213 A SINGLE CENTRE PROSPECTIVE STUDY ON FACTORS PREDICTING MALIGNANCY IN PATIENTS WITH DYSPEPSIA

${ }^{1}$ Anurag Shetty*, ${ }^{2}$ Ganesh Pai, ${ }^{2}$ Shiran Shetty, ${ }^{2}$ Girisha Balaraju, ${ }^{1}$ BV Tantry, ${ }^{2}$ Nagesh Kamath, ${ }^{1}$ Sandeep Gopal, 'Suresh Shenoy. ${ }^{1}$ KMC Mangalore, Manipal Academy of Higher Education, India; ${ }^{2}$ KMC Manipal, Manipal Academy of Higher Education, India

\subsection{6/gutjnl-2018-IDDFabstracts. 158}

Background Cultural, local dietary and social factors, the frequency of malignancy in the population studied are known to affect the epedimiology of dyspepsia. Clinical features are highly unreliable in determining the aetiology of dyspepsia. Alarm features are known to help in identifying underlying malignancy in patients with dyspepsia. However, very few studies are available from Asia validating the role of alarm features in dyspepsia.

Methods 900 consecutive patients with dyspepsia were included in the study after recording relevant clinical details such as symptoms, duration, age, weight and alarm features. All patients underwent standard upper gastrointestinal endoscopy, and the findings were recorded. All suspected malignancies were confirmed with histopathology. All malignancies and alarm features were statistically analysed using chi-square test for non-parametric data while ANOVA and students T-test were used for parametric data.

Results On endoscopy, malignant lesions were seen in $5.5 \%$ of our subjects whereas alarm features were present in $22.9 \%$ of our subjects. Among the malignant lesions, esophageal malignancy was seen in 2.2\%, gastric malignancy in $3.1 \%$ with the rest being a duodenal malignancy. All the alarm features put together had a sensitivity and specificity of $92 \%$ and $81.2 \%$ respectively for predicting malignancy. Highest sensitivity and specificity was for weight loss (76\%) and supraclavicular lymph nodes $(99.8 \%)$ respectively. Based on ROC curve the optimal age to begin malignancy screening was 46.5 years.

Conclusions Alarm features are a reasonable and effective tool for identifying upper gastrointestinal malignancies in patients with dyspepsia. Amongst all the alarm features weight loss is the best screening tool. The appropriate age to begin screening for malignancy appears to be 46.5 years.

\section{IDDF2018-ABS-0214 DIAGNOSTIC YIELD AND THERAPEUTIC POTENTIAL OF SINGLE BALLOON ENTEROSCOPY IN CHILDREN}

Pankaj Shrimal*, Mohan Ramchandani, Manohar Reddy, Zaheer Nabi, D Nageshwar Reddy. Asian Institute of Gastroenterology, India

\subsection{6/gutjnl-2018-IDDFabstracts. 159}

Background Evaluation of small bowel diseases has been revolutionised with the advent of capsule endoscopy and balloon assisted enteroscopy (BAE). As opposed to capsule endoscopy, $\mathrm{BAE}$ enables tissue biopsies and performing therapeutic procedures. BAE is an effective tool for the diagnosis and management of small bowel diseases in adults. However, data is limited in the paediatric population.

We aimed to evaluate the diagnostic yield and therapeutic potential of BAE in the paediatric population.

Methods The data of all the children under 18 years of age who underwent BAE with single balloon enteroscope (SBE) from August 2011 to December 2017 was analysed for the role of SBE in diagnosis and management of small bowel disease.

Results A total of 144 children (mean age 15.09 years; range 4-18 years, boys-96) underwent SBE during the study period. Indications were pain abdomen $(n=87)$, chronic diarrhoea $(n=33)$, obscure gastrointestinal bleeding (OGIB) $(n=19)$ and recurrent vomiting $(n=9)$. Oral enteroscopy was performed in 78 children with a mean insertion length of $183.35 \pm 62.51$ $\mathrm{cms}$ beyond the duodeno-jejunal flexure and mean procedure duration of $40.9 \pm 16.08 \mathrm{~min}$. Retrograde enteroscopy was performed in 66 children with a mean insertion length of 125.70 $\pm 45.24 \mathrm{cms}$ and mean duration of $42.04 \pm 10.05 \mathrm{~min}$. Diagnostic yield in children presenting with pain abdomen, chronic diarrhoea, OGIB and vomiting was 41.37\%, 44.82\%, 57.89\% and $22 \%$, respectively. In children with pain abdomen and significant weight loss, the diagnostic yield was $84.21 \%$. Therapeutic procedures were done in 8 children (polypectomy in 5 and argon plasma coagulation in 3), and 11 patients underwent surgery. In addition, four patients underwent intra-operative enteroscopy for OGIB, three underwent small bowel resection, one patient underwent gastro-jejunostomy for duodenal ulcer. No major complications were observed.

Conclusions SBE is a safe and effective tool for diagnosis and management of small bowel diseases in children. Pain abdomen was the most common indication for enteroscopy, and diagnostic yield was especially high in patients presenting with pain abdomen and associated significant weight loss.

\section{IDDF2018-ABS-0217 USEFULNESS OF ENDOSCOPIC ULTRASONOGRAPHY FEATURES IN PREDICTION OF MALIGNANT GASTRIC GASTROINTESTINAL STROMAL TUMOUR}

Wei Chih Sun*, Tzung Jiun Tsai, Ping I Hsu, Hoi Hung Chan, Kwok Hung Lai, Wen Chi Chen. Division of Gastroenterology and Hepatology, Department of Internal Medicine, Kaohsiung Veterans General Hospital, Taiwan

\subsection{6/gutjnl-2018-IDDFabstracts. 160}

Background Although many endoscopic ultrasonography (EUS) features have been introduced, preoperative determination of the malignant risk of gastric gastrointestinal stromal tumours (GISTs) is difficult. This study aimed to investigate whether EUS features can predict the malignant gastric GISTs.

Methods We retrospectively reviewed patients who had surgically resected gastric GISTs with high-risk EUS features such as non-oval shape, ulceration, irregular border, heterogeneity, echogenic foci, hypoechoic foci, cystic spaces, and calcification. The malignant risk of GISTs was based on the criteria of tumour size and mitotic count according to 2013 World Health Organisation classification and 2008 National Institutes of Health consensus. The correlations of high-risk EUS features with the malignant gastric GISTs were evaluated.

Results A total of 31 patients, including 20 males and 11 females, were enrolled. The mean age was $61.6 \pm 10.9$ years old. The mean tumour size was $3.4 \pm 2.9 \mathrm{~cm}$. With regards to the malignant risk, 7 (23\%) tumours had a very low risk, 10 $(32 \%)$ tumours had a low risk, $1(3 \%)$ tumour had a intermediate risk, 10 (32\%) tumours had a intermediate or high risk, and $3(10 \%)$ tumours had a high risk. Twelve (39\%) patients were diagnosed of malignant GISTs and had a larger proportion of tumour size $>2 \mathrm{~cm}(92 \%-53 \%)$, irregular border (75\%-26\%), and cystic change (33\%-0\%) than those with benign GISTs (p?0.05). In multivariate logistic regression 